\title{
Dynamic Processing in the Human Language System: Synergy between the Arcuate Fascicle and Extreme Capsule
}

\author{
Tyler Rolheiser, ${ }^{1}$ Emmanuel A. Stamatakis, ${ }^{2}$ and Lorraine K. Tyler ${ }^{1}$ \\ ${ }^{1}$ Centre for Speech, Language, and the Brain, Department of Experimental Psychology, University of Cambridge, Cambridge CB2 3EB, United Kingdom, and \\ ${ }_{2}^{2}$ Division of Anaesthesia, School of Clinical Medicine, University of Cambridge, Cambridge CB2 2QQ, United Kingdom
}

The production and comprehension of human language is thought to involve a network of frontal, parietal, and temporal cortical loci interconnected by two dominant white matter pathways. These two white matter bundles, often referred to as the dorsal and ventral processing tracts, are hypothesized to have markedly different language functions. The dorsal tract is thought to process phonological processing, while the ventral tract is considered to abet semantics. This proposed functional differentiation of tracts is similar to the ventral and dorsal dichotomy proposed for the visual and auditory systems. The present study evaluated this characterization of the language system in the context of various components involved in its function. Twenty-four chronic stroke patients completed a battery of 10 language tests designed to measure performance on the comprehension and production of phonology, morphology, semantics, and syntax. The patients also completed diffusion MRI scanning. Lesions were confined to the left hemisphere, but the size and location of the insult varied so that patients had damage to a single tract, both tracts, or neither tract. Individual FA maps were generated, and focal areas of hypointensity served as markers of white matter damage. Whole-brain voxel-by-voxel correlations revealed that only phonological and semantic tasks fit into the dual-stream model, while syntax and morphology involved both pathways. ROI analyses of the arcuate fascicle and extreme capsule supported this finding. These data suggest that natural language function is more likely to reflect a synergistic system rather than a segregated dual-stream system.

\section{Introduction}

Converging evidence suggests that the neural language network involves a left-lateralized, frontoparietotemporal network of gray matter regions subserved by major white matter pathways (Friederici et al., 2003; Hickok and Poeppel, 2004; Catani et al., 2005; Tyler and Marslen-Wilson, 2008). How these cortical and subcortical areas interact to produce a seamless behavioral system remains a major unanswered question. Central to this investigation is the contribution of the white matter tracts, with the primary focus on those directly connecting frontal and temporal regions - the arcuate fascicle (AF) and the extreme capsule (EmC) - the AF being the most recent to develop both phylogenetically and ontogenetically (Rilling et al., 2008; Brauer et al., 2011). Recent studies have quantified the two pathways in humans using diffusion tensor imaging (DTI) (Hua et al., 2008; Makris and Pandya, 2009). These analyses, together with nonhuman primate work (Petrides and Pandya, 2009), suggest that these two pathways are involved in the human language system.

\footnotetext{
Received June 1, 2011; revised Aug. 14, 2011; accepted Sept. 29, 2011.

Author contributions: E.A.S. and L.K.T. designed research; T.R. performed research; T.R., E.A.S., and L.K.T. analyzed data; T.R., E.A.S., and L.K.T. wrote the paper.

This research was supported by a Medical Research Council (UK) program grant to L.K.T. (Grant G0500842), and an ERC Advanced Investigator grant to L.K.T. We thank John Griffiths for constructing the tractography template, and Dr. Billi Randall and Caroline Jennings for help with the behavioral language tests.

The authors declare no competing financial interests.

Correspondence should be addressed to Prof. Lorraine K. Tyler, Centre for Speech, Language, and the Brain, Department of Experimental Psychology, University of Cambridge, Downing Street, Cambridge CB2 3EB, UK. E-mail: Iktyler@csl.psychol.cam.ac.uk.

DOI:10.1523/JNEUROSCI.2725-11.2011

Copyright $\odot 2011$ the authors $\quad 0270-6474 / 11 / 3116949-09 \$ 15.00 / 0$
}

One emerging conceptualization of the language system posits that the white matter tracts abet different forms of information processing (Hickok and Poeppel, 2007; Saur et al., 2008), with the AF involved primarily in phonology and/or articulation (Catani et al., 2005; Saur et al., 2008) and syntax (Friederici et al., 2003), and the EmC mainly implicated in semantics (Saur et al., 2008). While authors vary in their claims concerning the extent of functional specialization, all argue for some degree of functional differentiation. This functional characterization is supported by studies using functional MRI and DTI (e.g., Saur et al., 2008; Friederici et al., 2006; Friederici, 2009), leading to the proposal that the language system may be functionally subdivided, comparable to visual and auditory systems.

Despite the conceptual appeal of a dichotomous system, its operational nature remains speculative. No study has yet examined the two major white matter tracts in a broad language context, using tasks that sample a wide range of core language functions, such as phonology, morphology, syntax, and semantics. Measuring an extensive spectrum of language functions is necessary to evaluate how the pathways contribute to various aspects of language production and comprehension, and avoids the limitations of single-test experimental designs.

The present study represents a significant advance in characterizing the roles of the $\mathrm{AF}$ and the $\mathrm{EmC}$ in language by combining tract measures with behavioral data on a variety of language tests, to determine how white matter integrity relates to different aspects of language function. Twenty-four chronic stroke patients underwent DTI and behavioral testing. All patients had left-hemisphere damage, with variation in the size and location of 


\begin{tabular}{|c|c|c|c|c|}
\hline $\begin{array}{l}\text { Patient } \\
\text { a }\end{array}$ & Sex & Age & AF damage (\%) & EmC damage (\%) \\
\hline 1 & $M$ & 76 & 14.0 & 11.7 \\
\hline 2 & $M$ & 59 & 3.1 & 12.6 \\
\hline 3 & M & 63 & 47.0 & 67.4 \\
\hline 4 & M & 67 & 16.7 & 0.0 \\
\hline 5 & $M$ & 63 & 33.5 & 77.0 \\
\hline 6 & M & 41 & 11.8 & 0.0 \\
\hline 7 & $\mathrm{~F}$ & 34 & 5.6 & 0.0 \\
\hline 8 & $\mathrm{~F}$ & 70 & 42.9 & 0.0 \\
\hline 9 & M & 34 & 5.2 & 36.8 \\
\hline 10 & $M$ & 70 & 0.0 & 0.0 \\
\hline 11 & M & 55 & 76.2 & 90.4 \\
\hline 12 & M & 72 & 92.9 & 93.8 \\
\hline 13 & $\mathrm{~F}$ & 53 & 11.5 & 24.7 \\
\hline 14 & M & 46 & 88.2 & 92.1 \\
\hline 15 & M & 64 & 10.5 & 0.0 \\
\hline 16 & $\mathrm{~F}$ & 56 & 44.4 & 34.6 \\
\hline 17 & $\mathrm{~F}$ & 36 & 38.2 & 51.1 \\
\hline 18 & $\mathrm{~F}$ & 64 & 61.1 & 64.7 \\
\hline 19 & $\mathrm{~F}$ & 72 & 1.5 & 70.3 \\
\hline 20 & $\mathrm{~F}$ & 66 & 35.5 & 3.4 \\
\hline 21 & $\mathrm{~F}$ & 42 & 43.1 & 32.8 \\
\hline 22 & $\mathrm{~F}$ & 55 & 35.1 & 54.7 \\
\hline 23 & $\mathrm{~F}$ & 62 & 47.1 & 1.9 \\
\hline 24 & M & 61 & 47.4 & 1.3 \\
\hline Mean age & & 57.5 & & \\
\hline
\end{tabular}

The amount of damage to a given tract was calculated as a percentage of a tract that overlapped with a subject's lesion. A value of $100 \%$ would indicate that the entire tract overlapped with a lesion. Conversely, a value of $0 \%$ indicates relative sparing of that tract.

the lesion and in the extent to which one or other tract was damaged. Patients completed a battery of language tests spanning key aspects of language comprehension and production. Behavioral performance was correlated to diffusion measurements in wholebrain and region-of-interest analyses. This approach enabled us to determine whether, when considered across a broad range of language functions, the relationship between the AF and EmC is better described as a dual-stream system or a synergistic system.

\section{Materials and Methods}

Participants. Participants were recruited from the Centre for Speech, Language, and the Brain's panel of volunteers and from local stroke groups (Table 1). All participants (mean age 57.5 years; SD of 12.6 years) were at least 1 year removed from the cerebrovascular accident, with a median recovery interval of 3.5 years before testing (range from 1 to 21 years). In all cases, the stroke involved the left hemisphere exclusively, although the location and severity of the damage varied (Fig. 1). Before the stroke, all participants were dominantly right handed. Additional criteria for participant selection included the ability to provide informed consent, undertake task instructions, be native British English speakers, and have no contraindications to MRI scanning. A total of 24 patients ( $n=13$ males) completed testing after providing written consent (Suffolk Research Ethics Committee). All participants had normal audiometer measurements, and were not cognitively impaired as measured by the Mini-Mental State Examination (a score of 25 or higher) and/or above the 25th percentile on the Raven's Colored Progressive Matrices (total score of $26 / 36$ or better).

Behavioral tasks. Subjects performed a series of 10 behavioral tests designed to examine different aspects of language function (see Table 2 for summary of tests); these tests are used extensively in the Centre for Speech, Language, and the Brain. The testing was designed to measure speech comprehension and production at phonological, morphological, semantic, and syntactic levels.

MRI data collection and analysis. All MRI data were collected at the Medical Research Council Cognition and Brain Sciences Unit (MRC-
CBU, Cambridge), with a Siemens 3T Trio whole-body scanner (Siemens Medical Solutions). Two scans were collected. The first was a threedimensional MPRAGE T1-weighted sequence, with $1 \mathrm{~mm}$ isotropic resolution $(\mathrm{TR}=2250 \mathrm{~ms}$ and $\mathrm{TE}=2.99 \mathrm{~ms}$; acquisition time was $4 \mathrm{~min}$ $16 \mathrm{~s})$. The second pulse sequence was an EPI DTI sequence, collected along 64 orthogonal directions sampled twice. Salient collection parameters for this sequence are TR $=6600 \mathrm{~ms}, \mathrm{TE}=93 \mathrm{~ms}$, matrix $128 \times 128$, $3 \mathrm{~mm}$ slice thickness, and $\mathrm{b}$ values of 0 and $1000 \mathrm{~ms}$. The acquisition time was $12 \mathrm{~min}$.

All data were converted from dicom to nifti format using MRI convert 2.0 (Jolinda Smith, University of Oregon, Eugene, OR), and were visually checked for artifacts before analysis. All preprocessing was completed using FSL version 4.1.2 operating in a Linux environment. Converted data were preprocessed for eddy currents and subject motion using an affine registration model. Nonbrain tissue was removed using FSL's automated brain extraction tool (BET), and a diffusion tensor fitting model was then applied to the data. The diffusion tensor fitting provided estimates of fractional anisotropy (FA) and mean diffusivity (MD), as well as three principle eigenvectors and eigenvalues. These estimates were computed on individual voxels using a three-dimensional Gaussian distribution model that yielded a single mean ellipsoid for each voxel. The main variable of interest for this analysis was FA. Decreases in FA were interpreted as an analog of white matter tract damage.

Fractional anisotropy images were directly coregistered to standard space using a nonlinear registration protocol (FNIRT; Andersson et al., $2010 \mathrm{a}, \mathrm{b})$. The nonlinear standardization protocol uses both affinetransformation parameters (calculated using FLIRT) and nonlinear warps to register individual subjects' FA maps directly to FMRIB58 $1 \mathrm{~mm}$ isotropic space. This process was facilitated by creating custom masks of subjects' lesions. Including the masks in the nonlinear registration technique optimized the procedure by deweighting variance associated with sites of lesion (i.e., localized signal hypointensity). Lesion masks were hand traced on b0 images taken from the DTI scans. Spatial location of the lesion on the b0 image was confirmed via examination of the T1 image and cross-referenced with the neuroradiologist report. Lesions on the b0 images appeared as areas of signal hyperintensity, while on the T1 scans, lesion locations were characterized by signal dropout/areas of hypointensity. Finally, all spatially normalized images were smoothed using an $8 \mathrm{~mm}$ Gaussian FWHM kernel filter before statistical analysis.

The data were analyzed in two ways. The first analysis was performed using voxelwise whole-brain correlations between the scores on individual language tasks and subjects' FA maps. Each language task, therefore, produced a single brain map identifying those regions of the brain (if any) correlated with FA in our patient cohort. Criteria for significance were as follows: voxelwise correlation significance of $p<0.001$, with a cluster size significance of $p<0.05$, corrected for familywise error rate. This analysis was performed using a multiple regression model using SPM 8, run in Matlab (version 7.7).

We then focused on the two direct frontotemporal pathways of primary interest here using an ROI analysis. Two masks were created that represented the $\mathrm{AF}$ and the $\mathrm{EmC}$ in the left hemisphere (Fig. 2). To identify the white matter fibers associated with the AF, the Johns Hopkins University (JHU) white matter tractography atlas was used (displayed in FSLView). Using this atlas, we identified the white matter fibers contained within the temporal branch of the superior longitudinal fascicle (SLF). The SLF mask was then thresholded to contain only those voxels with an $80 \%$ probability (or greater) of inclusion in the SLF to eliminate voxels that likely would represent gray matter. The resulting mask, therefore, minimized the possibility of gray matter partial volume effects on the ROI data.

Since there were no available atlases that satisfactorily identified the EmC for an ROI analysis, the EmC mask was generated using a controlgroup tractography method. Briefly, nine healthy normal control subjects underwent the same DTI scanning protocol for the construction of the EmC tractography mask. A single seed was used to construct the deterministic pathway (Makris and Pandya, 2009). For the purpose of this study, the anterior termination point of the $\mathrm{EmC}$ was defined as the left inferior frontal gyrus (BA45) and the posterior termination point was defined as the left posterior middle temporal gyrus. All nine subjects had 


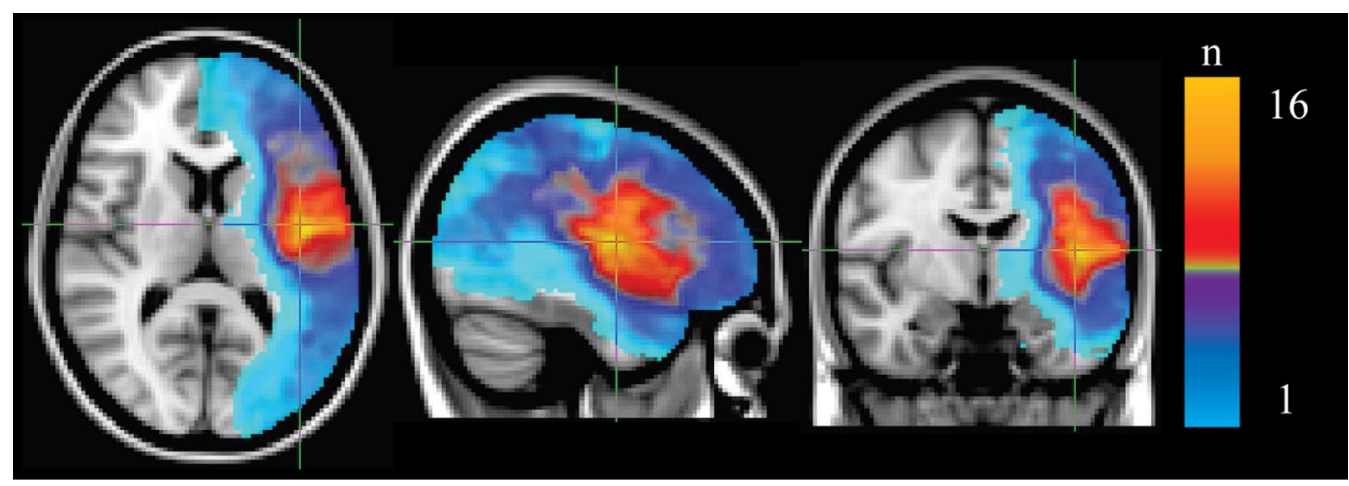

Figure 1. Frequency distribution plot of lesion location across all participants. Color map of lesion location overlaid on MN1152 standard space template. "Hot" colors (red, progressing to yellow) indicate that a greater number of subjects had lesions in that region. The crosshairs represent the peak region of subject lesion overlap, where 16 of a possible 24 subjects had detectable damage. Subjects were screened before scanning to ensure that the lesion location was confined exclusively to the left hemisphere.

Table 2. Language tests

\begin{tabular}{|c|c|c|c|}
\hline Category & Test name & Ref & Task \\
\hline \multicolumn{4}{|c|}{ Language comprehension } \\
\hline Phonology & PALPA segmentation & Kay et al., 1992 & $\begin{array}{l}\text { Hear a word or nonword. Listen for first or last sound and choose from a list of } 5 \text { sounds. } \\
\text { Measures sensitivity to phonological structure. Measure }=\% \text { correct. }\end{array}$ \\
\hline Morphology & Past tense judgments & $\begin{array}{l}\text { Adapted from Tyler et al., } \\
\text { 2002b, } 2005\end{array}$ & $\begin{array}{l}\text { Hear two words. Indicate whether they are the same or different. Words are stems and } \\
\text { regular or irregular past tenses (e.g. " "rinse"/"rinse"; "rinse"//"rinsed"; "sleep"/ } \\
\text { "slept"). Measures knowledge of morphological structure. Measure = \% correct. }\end{array}$ \\
\hline Syntax & Birkbeck Reversible Sentences & Tyler et al., 2002a, 2010, 2011 & $\begin{array}{l}\text { Hear a sentence and match to one of three pictures. Pictures are either correct or depict } \\
\text { a syntactic reverse role or a lexical distractor. Frequently selecting the picture with } \\
\text { the reverse role indicates insensitivity to syntax. Measure }=\% \text { error. }\end{array}$ \\
\hline Semantics & Property Knowledge & $\begin{array}{l}\text { Moss and Tyler, 2000; Bright } \\
\text { et al., } 2008\end{array}$ & $\begin{array}{l}\text { Respond to a set of questions about the properties of an object. Correct answers are } \\
\text { either yes ("Does a cat have whiskers?") or no ("Does a fork have skin?"). Measures } \\
\text { semantic knowledge. Measure }=\% \text { correct. }\end{array}$ \\
\hline
\end{tabular}

Language production Phonology

Segmentation task

Tyler et al., 2002b

Morphology

Past tense Elicitation: CSLB

Tyler et al., 2002a

Past Tense Elicitation: Ullman

Ullman et al., 1997

Semantics

Picture naming

Syntax

Sentence repetition
Bunn et al., 1998; Tyler et al., 2004

Adapted from Tyler et al., 2002a
Repeat a heard word without the final or initial phoneme (hear "Maid," then produce "may" or "aid"). Measures sensitivity to phonological structure. Measure $=\%$ correct.

Hear a sentence that ends with a verb, together with the beginning of a second sentence (e.g., "My nose sometimes bleeds. Last night, it ..." Produce a past tense form using verb in first sentence. Regular or irregular verbs are included. Measures ability to produce morphologically complex words. Measure $=\%$ correct.

Similar to above but with a nonsense verb (e.g., "plob") as an additional condition. Measure $=\%$ correct.

Name colored pictures of common objects. Measures ability to access semantic representations. Measure $=\%$ correct.

Repeat a set of short sentences taken from the Birkbeck Reversible sentences. Measures ability to produce syntactically well formed sentences. Measure $=\%$ correct.

PALPA, Psycholinguistic assessments of language processing in aphasia.

reconstructed fibers between these two regions via the EmC. Using these data, a probabilistic mask was generated whereby each voxel in the mask was assigned a value corresponding to how many subjects had a reconstructed fiber passing through it. The probabilistic EmC template was then coregistered to FMRIB58 standard FA space using an affine registration. These two masks were then used to extract FA values from patients to provide a measure of white matter tract integrity of the AF and EmC in the left hemisphere.

To determine how performance on a given language task covaried with FA of either ROI tract, a linear multiple regression was conducted (SPSS version 15.0 for Windows). All behavioral tasks were individually regressed by FA values associated with the AF and the EmC. Pearson's $r$, and squared semipartial correlation (SSC) coefficients were generated. By using these two measures to describe the data, it is possible to evaluate how each white matter pathway varies both generally $(r)$ and uniquely
(SSC) with a language task. As a final descriptor of these data, SSC values were used to compute a novel index that describes how language preferentially loads onto the two white matter pathways. This index is calculated as follows: Anatomical Loading $=[(\mid$ SSC AF $\mid)-(\mid$ SSC EmC $\mid)] \times$ 100. A higher score on the anatomical loading index (ALI) indicates a greater reliance on AF integrity, while a lower score suggests a greater reliance on EmC integrity.

\section{Results}

\section{Voxel-based correlations}

The behavioral data and MRI data were first analyzed for the possible confounding effect of age and basic cognitive processing (measured by the MMSE and Raven's Colored Matrices tests). Whole-brain, voxelwise correlations were performed on these 


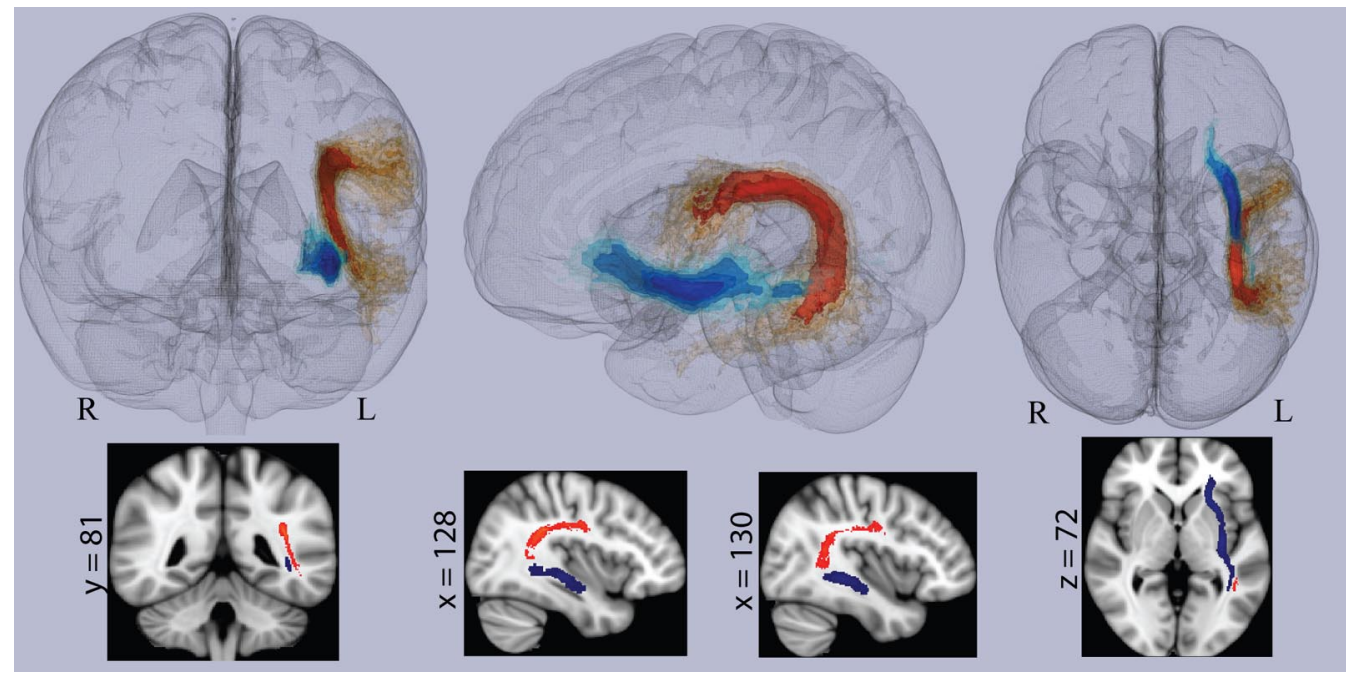

Figure 2. Regions of interest masks for the arcuate fascicle and the extreme capsule. The red/yellow mask is a probabilistic Rol mask representing the arcuate fascicle (temporal branch) as determined by the JHU tractography atlas. The blue/light blue mask represents a probabilistic ROI mask of the EmC, defined using standard tractography procedures in healthy normal control subjects. This figure also displays individual slices illustrating the spatial relationship between the AF and EmC near MTG. $x, y, z$ dimensions are shown in MNI152 $1 \mathrm{~mm}$ space.

three measures individually to identify possible clusters of white matter that significantly correlated with these variables. No significant clusters emerged from these correlation analyses. As an additional control, we ran correlations between global FA, age, and performance on the Raven's task and the 10 language tasks. All of the Pearson $r$ values for these three measures were $<0.227$, and none approached significance (all $p>0.331$ ).

Using the whole-brain, voxel-based correlation analysis, 9 of the 10 language tests produced clusters of significance using the voxel significance level of $p<0.001$ and an FWE-corrected cluster significance level of $p<0.05$. However, although the semantic production test of picture naming did not reach this level of significance, performance did significantly correlate with FA at a voxel significance level of $p<0.01$, and the same FWE-corrected cluster level of $p<0.05$. Given the frequent use of picture naming in studies of language production, we include it here. Summary statistics and anatomical locations of the clusters can be found in Figures 3 (comprehension tasks) and 4 (production tasks).

\section{Language comprehension tasks}

The clusters associated with tests of language comprehension varied with each task, such that a cluster could be located on the dorsal tract, the ventral tract, or some combination thereof. All effects were located in the left hemisphere. Behavioral performance on the two tests of comprehension phonology were highly correlated $(r=0.802, p<0.001)$, and so a mean score was computed and used in the whole-brain correlation analysis. Performance on two language tasks (phonology and morphology) correlated with FA in the dorsal pathway, one (semantics) correlated with FA in the ventral pathway, and one (syntax) correlated with FA in both pathways. The correlation between FA and comprehension phonology produced a significant cluster of voxels centered on the anterior portion of the AF, extending anteriorly toward the precentral gyrus and posteriorly toward the supramarginal gyrus. Similarly, performance on comprehension of past tense morphology correlated with FA only within the dorsal tract, with the cluster overlapping with the SLF near BA 39/BA 40. This effect was largely due to regularly inflected words. Performance on the irregular past tense did not significantly correlate with any voxels, undoubtedly due to the fact that most patients made very few errors on this test. The results for the semantic test showed that performance correlated with FA in the ventral pathway. The significant cluster was contained within the posterior segment of the EmC, in close proximity to the posterior middle temporal gyrus. Comprehension of syntax produced two significant correlation clusters centered on both tracts. One was predominantly centered on the posterior portion of the AF and extended superiorly toward the supramarginal gyrus, and the other was located in the white matter of the temporal pole, and extended superiorly toward the IIFG.

\section{Language production tasks}

Performance on the language production tasks revealed a broadly similar relationship between language function and white matter pathway as observed with the comprehension tests. FA in the dorsal pathway was associated with performance on the phonology task, with the significant cluster extending from the posterior MTG toward the postcentral gyrus along the anterior AF. The dorsal pathway alone was also involved in performance on inflectional morphology tests, but only when the to-be-inflected words were nonsense verbs. Production of nonwords was associated with the anterior AF exclusively. In contrast, production of morphologically complex real words was associated with two significant clusters: The first, situated anteriorly, involved the EmC at the inferior margin and the anterior AF nearer the superior border. The second cluster extended from the MTG, following the course of the posterior AF, and terminated adjacent to the postcentral gyrus. Since only real words by definition contain semantics, this highlights the relationship between semantics and the ventral pathway. This relationship was confirmed by the results of the semantics task that produced a single significant correlation (at the slightly more liberal voxel-level threshold $p<0.01$ ) located in the anterior segment of the ventral pathway, overlapping with fibers from the EmC.

Finally, performance on syntax production was associated with two significant clusters. The larger posterior cluster overlapped predominantly with the entire AF but shared some overlap with the EmC in the inferior portion of the cluster near the 
Peak Coordinate

$\begin{array}{lllllll}\text { Task } & \text { Cluster Sig. } & X & Y & Z & \text { Cluster Morphology } & \text { Behavioural Scatterplot }\end{array}$

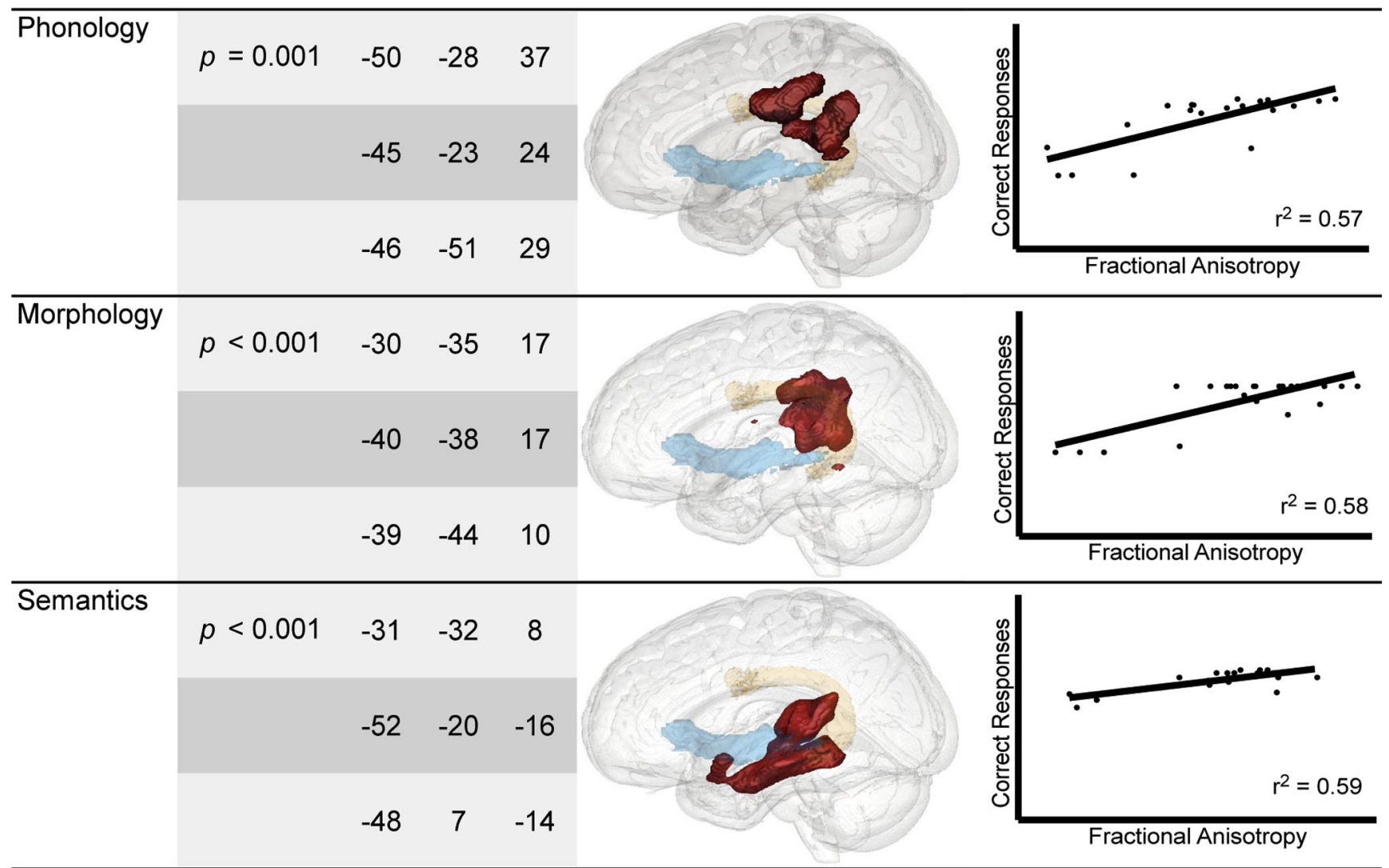

Syntax:

Anterior

$p<0.001 \quad-22 \quad 26 \quad-10$

Cluster

$\begin{array}{lll}-28 & 32 & 0 \\ -27 & 35 & 18\end{array}$
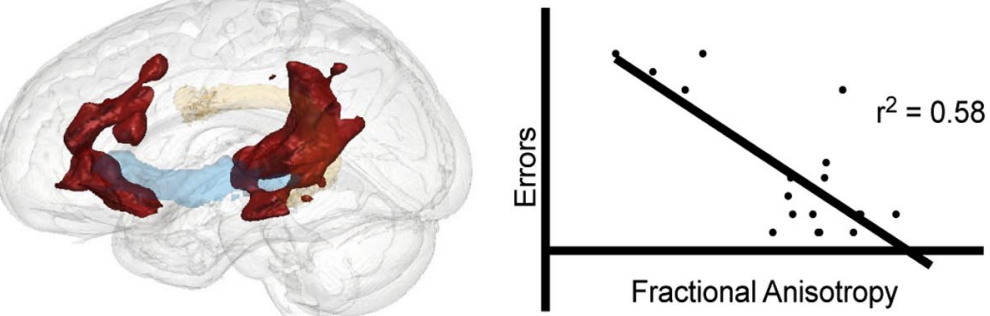

Fractional Anisotropy

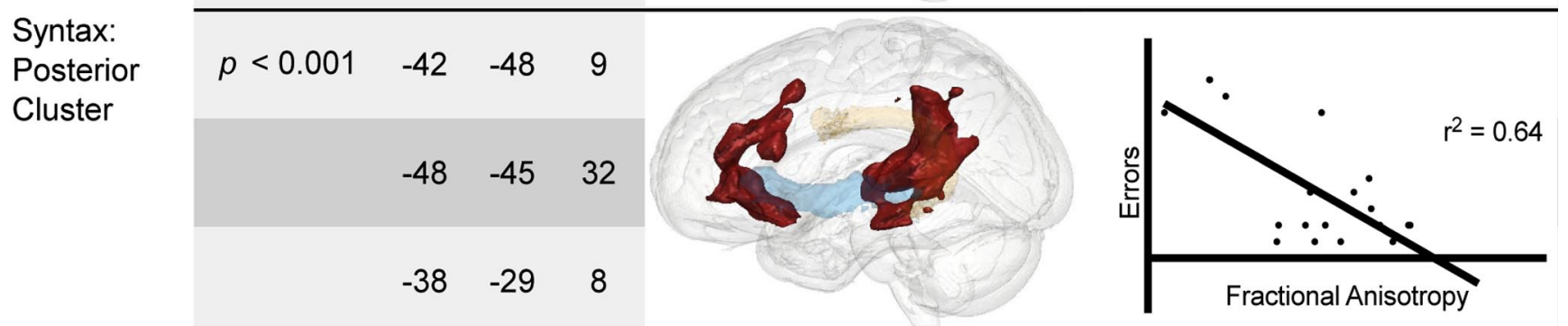

Figure 3. Whole-brain correlations between comprehension tasks and fractional anisotropy. Shown are results from the whole-brain voxelwise correlation analysis between language comprehension tasks and FA. Each significant cluster is described in terms of significance level, and peak location of the three highest voxels in MNI152 space. The significant cluster itself can be seen on the three-dimensional rendering in the middle column. We show the arcuate fascicle in orange (yellow) and the extreme capsule in blue. The plots on the far right show subjects' performance against FA values extracted from the cluster.

MTG. The second significant cluster involved the EmC inferiorly and the anterior AF superiorly. These data imply a certain propensity for some tasks to be biased toward the dorsal or ventral pathway. Despite this bias, performance of some of the tasks also correlated with both the AF and the EmC.
Lesion location: overlap with the $\mathrm{AF}$ and $\mathrm{EmC}$

Individual subject lesion masks were analyzed for the amount (percentage) of overlap with both the AF and the EmC ROI masks. All lesion masks were confined to the left hemisphere. This provided a measure of the extent of damage present in either 
Peak Coordinate

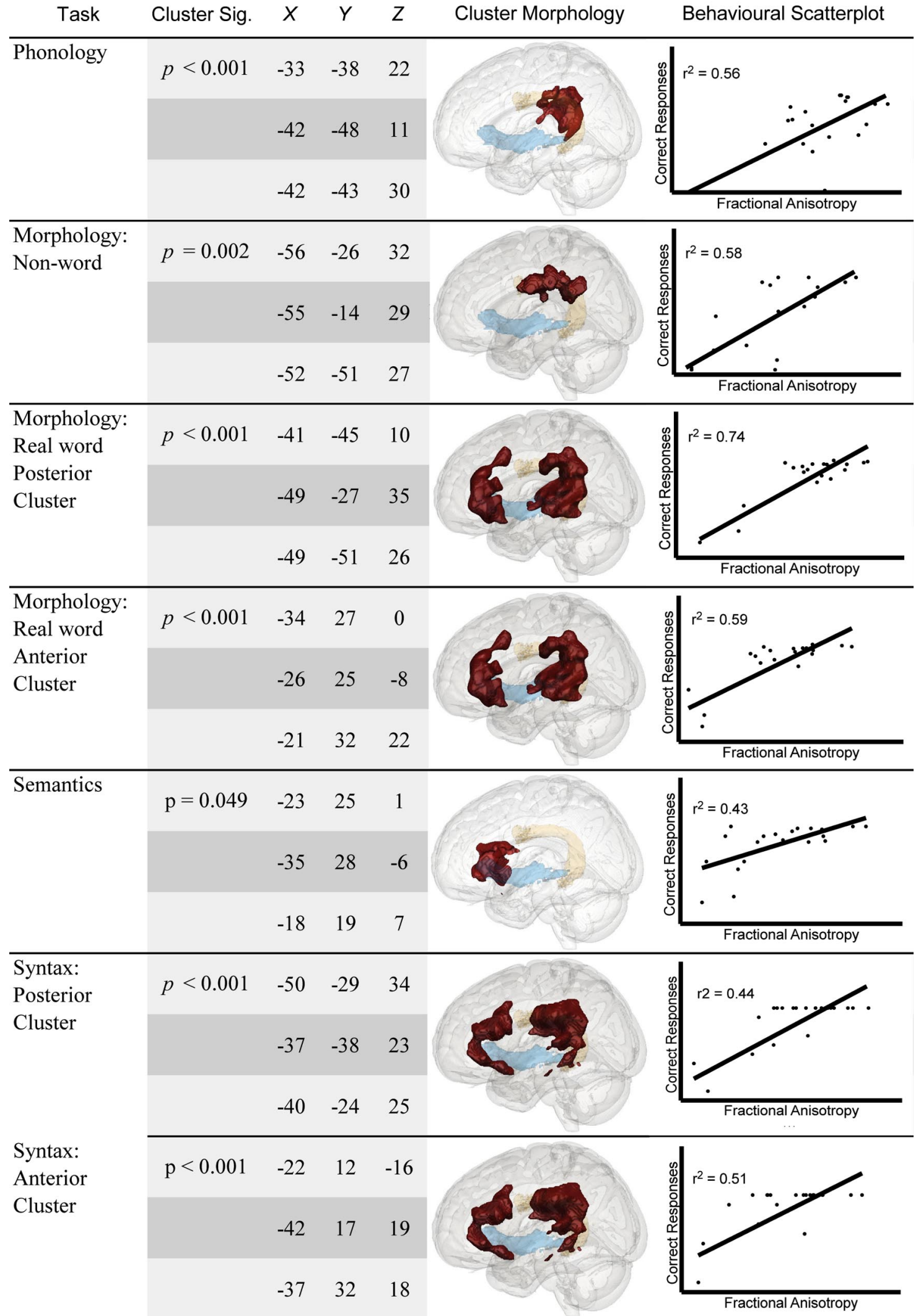

Figure 4. Whole-brain correlations between production tasks and fractional anisotropy. Shown are results from the whole-brain voxelwise correlation analysis between language production tasks and FA. Each significant cluster is described in terms of significance level, and peak location of the three highest voxels in MNI152 space. The significant cluster itself can be seen on the three-dimensional rendering in the middle column in red. We show the arcuate fascicle in orange (yellow) and the extreme capsule in blue. Plots on the far right show subjects' performance against FA values extracted from the cluster. 
Damage to the AF and EmC: Variability Plot

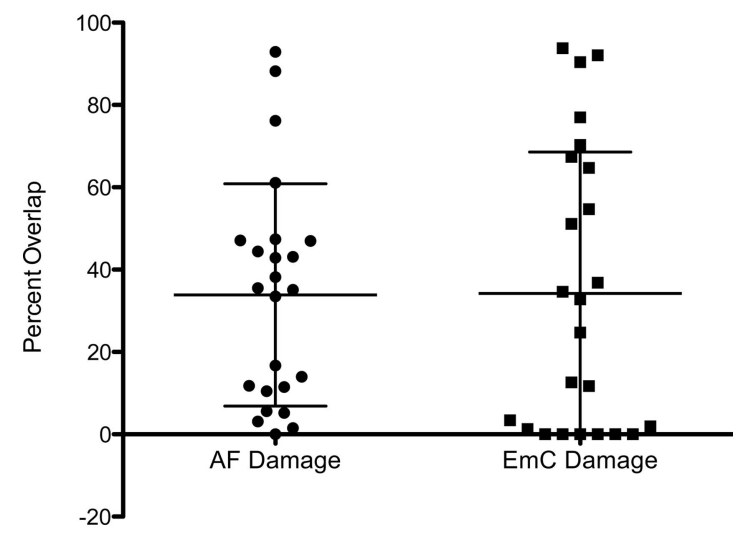

Figure 5. Lesion variability plot. Shown is the range of damage in the AF and the EmC across all subjects. Each subjects' lesion map was compared to the AF and EmC ROls, and the degree of overlap was computed. The middle horizontal bar represents the mean overlap value, with the error bars representing SD.

white matter tract across all subjects (Fig. 5). In both cases, the amount of damage to the $\mathrm{AF}$ and EmC existed along a large continuum such that some patients had no white matter damage to a given tract, while others had nearly complete damage to a particular tract. The overlap distribution did not display significant skewness or kurtosis for either the $\operatorname{AF}(z=1.41 ; z=-0.23)$ or the EmC $(z=1.06 ; z=1.39)$ and thus could be considered normally distributed.

\section{Anatomical loading data}

Since our main interest was in the functional roles of the two direct frontotemporal pathways - the $\mathrm{AF}$ and EmC fiber bundles - we analyzed the language data for unique variance between behavior and FA of the AF and EmC using the tract ROIs. SSCs were computed between the FA and all language tests by removing all common variance between the $\mathrm{AF}$ and $\mathrm{EmC}$, and then calculating a correlation between a single white matter tract and the behavioral tasks. These SSCs were used to construct an anatomical loading index that orders language tasks by the amount of unique variance between a language task and the AF or EmC (Fig. 6). This index represents a measure of the strength of association between the language tasks and the AF and EmC. Scores at the top of the $y$-axis are the tasks that are associated most strongly with the integrity of the AF. Moving toward the bottom on the $y$-axis are tasks that rely more heavily on the integrity of the EmC. When the language tasks were ordered according to unique variance with the $\mathrm{AF}$ and $\mathrm{EmC}$, we see that semantic tests consistently load on the EmC. The loading of other tests, however, varies as a function of the specific language test and whether subjects are performing comprehension or production tasks. The tests and tasks that load most heavily on the AF are syntactic, morphological, and phonological comprehension and the production of inflected nonwords. The behavioral variables clustered around the zero point on the $y$-axis (i.e., balanced reliance on both tracts) are the comprehension of syntax and the production of morphologically complex real words.

\section{Discussion}

We examined 24 chronic stroke patients who had suffered left hemisphere damage using DTI and behavioral language testing. Using a whole-brain voxelwise correlation analysis, a variety of language test scores covering production and comprehension of phonology, morphology, semantics, and syntax significantly correlated with FA. In the whole-brain analyses, these clusters were located in the dorsal and ventral pathways in the left hemisphere, consistent with anatomical models of language underpinned by these two frontotemporal white matter bundles. To specifically evaluate the functional roles in language of the two direct frontotemporal tracts - the AF and EmC fiber bundles-we used an ROI approach in which masks of each tract were constructed. This enabled us to determine the relative contribution of each tract to different aspects of language function in both comprehension and production.

The present results provide a broad, comprehensive perspective on the contribution of white matter to language function. Significantly, these data depart from the concept of a dorsal/ ventral separation of function in all but the most clearly defined phonological and semantic tasks. In both production and comprehension, phonological processing loaded most heavily on the AF such that most of the unique variance was accounted for by its relationship with the $\mathrm{AF}$, and semantic tasks loaded most heavily on the EmC. These results are consistent with current models of strongly differentiated pathways, with the AF involved in sensory-motor mapping (Hickok and Poeppel, 2007; Glasser and Rilling, 2008; Saur et al., 2008) and phonological processing (Catani et al., 2005) and the EmC involved with semantics (Catani et al., 2005; Glasser and Rilling, 2008). Syntax and morphology, on the other hand, showed a different pattern of correlations in which each type of linguistic function was associated with the AF alone under some task conditions and with both tracts under others.

In the whole-brain correlation analysis between morphological processing and FA, the comprehension of regularly inflected past tense forms was associated only with the integrity of the dorsal fiber bundle, consistent with claims for dorsal route involvement in the processing of regularly inflected past tense words (Marslen-Wilson and Tyler, 2007). Moreover, the results of the anatomical loading analysis showed that the EmC was only marginally involved, suggesting that sensitivity to the morphological structure of regularly inflected words during language comprehension may only minimally involve semantics. The production of morphologically complex nonwords also only involved the integrity of the AF and not the EmC. Since nonwords have minimal semantics, this supports claims for the role of the EmC in semantic aspects of language function. The production of morphologically complex real words, in contrast, shows a more balanced loading on both tracts, suggesting that this linguistic function involves access both to the semantics of the stem and the syntactic properties of the affix (Tyler and Marslen-Wilson, 2008).

The whole-brain correlations showed that both dorsal and ventral bundles were associated with syntactic performance in both comprehension and production tasks. However, when we focused on the two direct frontotemporal tracts in the ROI analysis, the specific involvement of the $\mathrm{AF}$ and the $\mathrm{EmC}$ in syntactic processing was differentiated according to whether the task was production or comprehension. Comprehending syntax loaded equally on both the $\mathrm{AF}$ and the $\mathrm{EmC}$, supporting recent data from tractography analyses (Griffiths et al., 2009). This finding emphasizes the critical role of these two pathways in linking the left frontal and middle temporal regions known to be involved in syntactic comprehension (Rodd et al., 2010). Damage to either cortical region or the connectivity between them causes syntactic deficits (Tyler et al., 2011). 
Syntactic production, in contrast, loaded most strongly on the AF, perhaps reflecting the specific properties of the syntactic production task used here, which involved repeating sentences and where performance is measured according to whether the syntactic structure of the original sentence is preserved. This is a standard test used to assess syntactic production abilities in aphasic patients (Caramazza and Hillis, 1989). It remains to be seen whether production tasks that involve a greater emphasis on the spontaneous generation of syntactic structures in production would show a greater loading on the EmC.

These findings highlight the importance of considering the range of linguistic components when evaluating the functional role of the white matter tracts in language function. We suggest that normal language function requires that information be transmitted between lateral prefrontal and posterior temporal cortical regions from both white matter tracts. In this way, the overall determinant of task performance is not dictated by which white matter tract is involved, but by how cortical regions use and integrate a constant flow of different kinds of linguistic information. This view of the white matter scaffolding holds additional appeal due to the anatomical convergence of the tracts at the MTG, and the common anterior projection of the AF and EmC toward the left IFG (Turken and Dronkers, 2011). By defining the white matter architecture as a synergy, the overall determinant of task performance is not dictated by which white matter tract is "involved," but by how cortical regions self-organize to produce a very complex behavior.

\section{A model of white matter tracts: branching out}

The present study is consistent with most published reports in its approach to modeling the white matter foundations of language (Friederici et al., 2003; Hickok and Poeppel, 2004; Catani et al., 2005; Saur et al., 2008). Two white matter pathways were identified using either well known atlas tools (AF) or an established deterministic tractography procedure (EmC). Together, these two tracts are considered the major pathways linking the temporal, parietal, and lateral prefrontal cortices. Their combined function is to reciprocally connect gray matter loci, thereby establishing temporally and spatially defined networks that determine function (Hickok and Poeppel, 2007). It is important to underscore the idea that white matter itself does not produce behavior. White matter provides the framework for communication in a system relying on gray matter. Because of this relationship, it becomes problematic to ascribe functions to individual white matter tracts (such as dorsal and ventral). Additionally, evidence suggests that the cerebral language network is subserved by tracts other than simply the AF and the EmC.

In a detailed examination of the AF, Catani et al. (2005) examined the white matter connections between Broca's and Wernicke's areas (see also Galuntucci et al., 2011). This analysis was prompted by the clinical variability of conduction aphasia seen in patients with lesions to the AF. Using DTI in 11 healthy normal controls, it was established that in addition to the classical direct pathway, an indirect pathway could also be defined within the AF. This second pathway linked Broca's and Wernicke's areas through Geschwind's territory, at the parietal-temporal-frontal junction. Within the AF, therefore, it is possible to resolve multiple gray-white matter interfaces necessary for natural linguistic functioning. These empirical data outline the complex nature of the white matter tracts presently under investigation, and caution against ascribing hardwired functions to such large white matter groups.

Similar examinations have been performed on the white matter normally associated with the ventral processing stream. In a

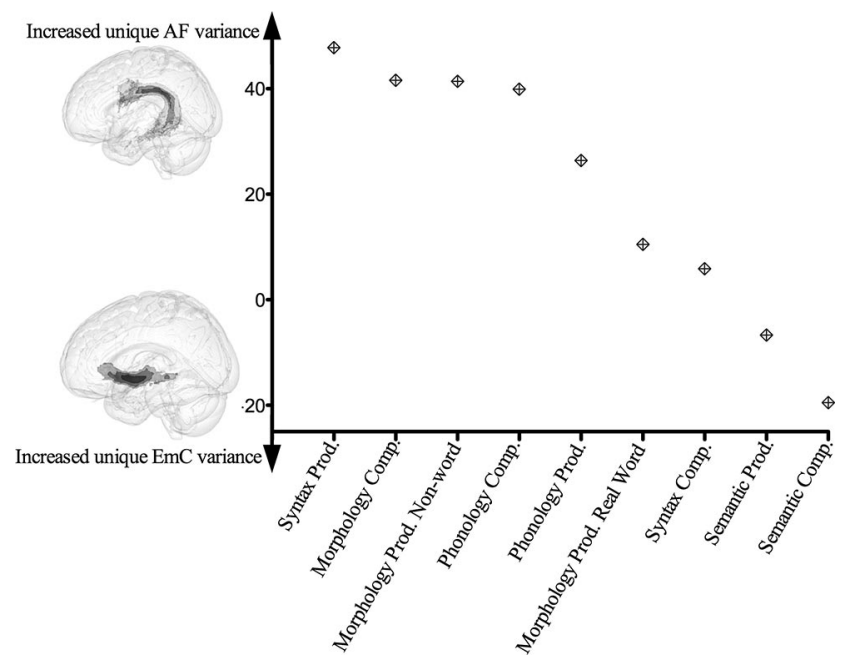

Figure 6. Unique variance between behavioral tasks and the two white matter tracts. Behavioral tests were arranged along a continuum representing the amount of unique variance between $\mathrm{FA}$ and the behavioral tests. Tasks that have more unique variance with the AF are toward the top, while those tasks that have more unique variance with the EmC appear toward the bottom. The zero point on the $y$-axis represents an equal amount of unique variance for both the $\mathrm{EmC}$ and the $\mathrm{AF}$ and a given behavioral task.

series of two studies, Mandonnet et al. (2007), and Duffau et al. (2009) used intraoperative direct electrostimulation to examine how white matter bundles near the EmC contributed to semantic tasks. In both cases, the authors propose the existence of a direct white matter pathway that subserves semantic tasks (EmC), and an indirect pathway (uncinate) that contributes to but is not necessary for language function. These results point to a very broadly interconnected model of white matter organization surrounding the ventral white matter pathway. Considered in tandem with the direct and indirect pathways through the AF, the dorsal and ventral language pathways may be served by more than two direct temporal-frontal connections. Given this likely scenario, a fruitful way forward in understanding the relationship between white matter function and language would be to focus on how different white matter pathways interact with multiple gray matter foci, rather than how to ascribe function to individual white matter tracts.

\section{Conclusion}

Using a combination of neuropsychological inference, DTI, and comprehensive language testing, this study demonstrated that involvement of either the AF or the EmC is contingent upon the varying demands of different components of language function. These results extend our current understanding of the contribution of white matter to language by documenting how performance of phonology, morphology, semantics, and syntax make use of communicating pathways. The observed relationship between white matter and behavior displays task-specific variability, and challenges the simple model of processing streams based purely on phonology and semantics respectively. Taken in concert with recent studies documenting indirect and secondary white matter pathways relevant to language, it is likely that relevant frontotemporal language connections operate as a synergy. Ultimately, behavior emerges as a product of coordinated gray matter activity; this necessitates the appropriate understanding and definition of the underlying white matter framework.

\section{References}

Andersson JLR, Jenkinson M, Smith S (2010a) Non-linear optimisation. FMRIB technical report TR07JA1. 
Andersson JLR, Jenkinson M, Smith S (2010b) Non-linear registration, aka spatial normalisation. FMRIB technical report TR07JA2.

Brauer J, Anwander A, Friederici AD (2011) Neuroanatomical prerequisites for language functions in the maturing brain. Cereb Cortex 21:459-466.

Bright P, Moss HE, Stamatakis EA, Tyler LK (2008) Longitudinal studies of semantic dementia: the relationship between structural and functional changes over time. Neuropsychologia 46:2177-2188.

Bunn EM, Tyler LK, Moss HE (1998) Category-specific semantic deficits: the role of familiarity and property type reexamined. Neuropsychology 12:367-379.

Caramazza A, Hillis AE (1989) The disruption of sentence production: some dissociations. Brain Lang 36:625-650.

Catani M, Jones DK, ffytche DH (2005) Perisylvian language networks of the human brain. Ann Neurol 57:8-16.

Duffau H, Gatignol P, Moritz-Gasser S, Mandonnet E (2009) Is the left uncinate fasciculus essential for language? A cerebral stimulation study. J Neurol 256:382-389.

Friederici AD (2009) Pathways to language: fiber tracts in the human brain. Trends Cogn Sci 13:175-181.

Friederici AD, Rüschemeyer S-A, Hahne A, Fiebach CJ (2003) The role of left inferior frontal and superior temporal cortex in sentence comprehension: localizing syntactic and semantic processes. Cereb Cortex 13:170-177.

Galantucci S, Tartaglia MC, Wilson SM, Henry ML, Filippi M, Agosta F, Dronkers NF, Henry RG, Ogar JM, Miller BL, Gorno-Tempini ML (2011) White matter damage in primary progressive aphasias: a diffusion tensor tractography study. Brain 34:3011-3029.

Glasser MF, Rilling JK (2008) DTI tractography of the human brain's language pathways. Cereb Cortex 18:2471-2482.

Griffiths JD, Stamatakis EA, Tyler LK (2009) Damage to dorsal and ventral frontotemporal white matter pathways impairs syntactic aspects of language comprehension: a DTI tractography study. Neuroimage 47:S143.

Hickok G, Poeppel D (2004) Dorsal and ventral streams: a framework for understanding aspects of the functional anatomy of language. Cognition 92:67-99.

Hickok G, Poeppel D (2007) The cortical organization of speech processing. Nat Rev Neurosci 8:393-402.

Hua K, Zhang J, Wakana S, Jiang H, Li X, Reich DS, Calabresi PA, Pekar JJ, van Zijl PC, Mori S (2008) Tract probability maps in stereotactic spaces: analyses of white matter anatomy and tract-specific quantification. Neuroimage 39:336-347.

Kay J, Lesser R, Coltheart M (1992) Psycholinguistic assessments of language processing in aphasia (PALPA). London: Lawrence Erlbaum.

Makris N, Pandya DN (2009) The extreme capsule in humans and rethinking of the language circuitry. Brain Struct Funct 213:343-358.

Mandonnet E, Nouet A, Gatignol P, Capelle L, Duffau H (2007) Does the left inferior longitudinal fasciculus play a role in language? A brain stimulation study. Brain 130:623-629.
Marslen-Wilson WD, Tyler LK (2007) Morphology, language and the brain: the decompositional substrate for language comprehension. Philos Trans R Soc Lond B Biol Sci 362:823-836.

Moss HE, Tyler LK (2000) A progressive category-specific semantic deficit for non-living things. Neuropsychologia 38:60-82.

Petrides M, Pandya DN (2009) Distinct parietal and temporal pathways to the homologues of Broca's area in the monkey. PloS Biol 7:e1000170.

Rilling JK, Glasser MF, Preuss TM, Ma X, Zhao T, Hu X, Behrens TEJ (2008) The evolution of the arcuate fasciculus revealed with comparative DTI. Nat Neurosci 11:426-428.

Rodd JM, Longe OA, Randall B, Tyler LK (2010) The functional organization of the fronto-temporal language system: evidence from syntactic and semantic ambiguity Neuropsychologia 48:1324-1335.

Saur D, Kreher BW, Schnell S, Kümmerer D, Kellmeyer P, Vry MS, Umarova R, Musso M, Glauche V, Abel S, Huber W, Rijntjes M, Hennig J, Weiller C (2008) Ventral and dorsal pathways for language. Proc Natl Acad Sci U S A 105:18035-18040.

Turken AU, Dronkers NF (2011) The neuronal architecture of the language comprehension network: converging evidence from lesion and connectivity analyses. Front Syst Neurosci 5:1-20.

Tyler LK, Marslen-Wilson W (2008) Fronto-temporal brain systems supporting spoken language comprehension. Philos Trans R Soc Lond B Biol Sci 363:1037-1054.

Tyler LK, deMornay-Davies P, Anokhina R, Longworth C, Randall B, Marslen-Wilson WD (2002a) Dissociations in processing past tense morphology: neuropathology and behavioural studies. J Cogn Neurosci 14:79-94.

Tyler LK, Randall B, Marslen-Wilson WD (2002b) Phonology and neuropsychology of the English past tense. Neuropsychologia 40:1154-1166.

Tyler LK, Stamatakis EA, Bright P, Acres K, Abdallah S, Rodd JM, Moss HE (2004) Processing objects at different levels of specificity. J Cogn Neurosci $16: 351-362$.

Tyler LK, Stamatakis EA, Post B, Randall B, Marslen-Wilson W (2005) Temporal and frontal systems in speech comprehension: an fMRI study of past tense processing. Neuropsychologia 43:1963-1974.

Tyler LK, Wright P, Randall B, Marslen-Wilson WD, Stamatakis EA (2010) Reorganisation of syntactic processing following LH brain damage: does RH activity preserve function? Brain 133:3396-3408.

Tyler LK, Marslen-Wilson WD, Randall B, Wright P, Devereux BJ, Zhuang J, Papoutsi M, Stamatakis EA (2011) Left inferior frontal cortex and syntax: function, structure and behaviour in left-hemisphere damaged patients. Brain 134:415-431.

Ullman MT, Corkin S, Coppola M, Hickok G, Growdon JH, Koroshetz WJ, Pinker S (1997) A neural dissociation within language: evidence that the mental dictionary is part of declarative memory, and that grammatical rules are processed by the procedural system. J Cogn Neurosci 9:266-276 